\title{
气载钎剂辅助钢/铝激光熔钎焊工艺与组织
}

\author{
喻高扬陈树海 黄继华杨 健 赵志毅 \\ (北京科技大学材料科学与工程学院 北京 100083)
}

\begin{abstract}
摘要: 为解决钢/铝异种金属熔钎焊过程中液态金属铝在固态钢表面润湿性差的问题, 提出一种气载钎剂辅助钢/铝异种金属 激光熔钎焊的新方法。采用不锈钢与 5052 铝合金作为母材, 分别进行激光填丝与无丝搭接熔钎焊试验。研究焊接线能量对 接头润湿角、接头力学性能的影响, 分析有无焊丝情况下接头典型微观组织。结果表明, 气载钎剂辅助不锈钢/铝合金异种金 属激光熔钎焊能有效解决液态金属在不锈钢表面的润湿铺展性问题。填丝搭接熔钎焊得到的接头润湿角最小值为 $37^{\circ}$, 接头 拉伸强度最大达到 $132.8 \mathrm{MPa}$, 约为铝合金强度 $(211.7 \mathrm{MPa})$ 的 $62.7 \%$; 无丝搭接熔钎焊得到的接头润湿角最小值为 $18.8^{\circ}$, 接 头拉伸强度最大达到 $109.2 \mathrm{MPa}$, 约为铝合金强度的 $51.6 \%$ 。在填丝、无丝焊接时，钢侧界面均会形成 $\mathrm{Fe}_{2} \mathrm{Al}_{5}$ 与 $\mathrm{FeAl}_{3}$ 两层金 属间化合物(Intermetallic compounds, IMCs)。
\end{abstract}

关键词: 钢/铝连接; 激光熔钎焊; 焊接工艺; 微观组织

中图分类号: TG456

\section{Technology and Microstructure for Laser Welding-brazing Assisted by Transporting Flux with Shielding Gas between Steel and Aluminum}

\author{
YU Gaoyang CHEN Shuhai HUANG Jihua YANG Jian ZHAO Zhiyi \\ (School of Materials Science and Technology Beijing, \\ University of Science and Technology Beijing, Beijing 100083)
}

\begin{abstract}
In order to solve the problem of poor wettability of liquid metal on the surface of stainless steel, a new method of powder flux transported by shielding gas is proposed to assist laser welding-brazing process. Laser welding-brazing of 5052 aluminum alloy and stainless steel with/without filler wire is carried out. Influence of welding heat input on wetting angle and mechanical property is studied. The typical microstructure of the joint with/without filler wire is analyzed. The results show that the laser welding brazing assisted by transporting flux with shielding gas for stainless steel/aluminum alloy dissimilar metals could improve wettability of liquid metal on stainless steel effectively. When the filler wire is used, the wetting angle of the joint is 37 degree. The tensile strength of the joint is about $132.8 \mathrm{MPa}$, which is about $62.7 \%$ of the strength of the aluminum alloy (211.7 MPa). In absence of filler wire, the wetting angle of the joint is 18.8 degree. The tensile strength of the joint is about $109.2 \mathrm{MPa}$, which is about $51.6 \%$ of the strength of the aluminum alloy. Two intermetallic compounds $\left(\mathrm{Fe}_{2} \mathrm{Al}_{5}\right.$ and $\left.\mathrm{FeAl}_{3}\right)$ will be form at the steel side interface with or without filler wire.
\end{abstract}

Key words: steel/aluminum joining; laser welding-brazing; welding technology; microstructure

\section{0 前言}

全球气候的持续变暖和能源的日益短缺, 环境 污染逐渐加重, 使得节能减排已成为世界各国的普 遍共识。产品的轻量化是实现节能减排的重要途径。 铝合金具有质轻、易加工、耐腐蚀等优点, 在汽车

* 国家自然科学基金(51875037)、北京市自然科学基金(3192021)和中央高 校基本科研业务(FRF-GF-18-004B)资助项目。20190811 收到初稿, 20190927 收到修改稿
制造中使用铝合金代替部分钢，可以有效实现产品 的轻量化, 因此, 钢和铝的连接在汽车制造等领域 具有广阔的应用前景 ${ }^{[1-3]}$ 。

钢/铝焊接的核心问题在于焊接过程中在钢侧界 面产生脆性金属间化合物, 这些金属间化合物具有较 高的脆性与较低的塑性, 是影响接头力学性能的关键 ${ }^{[4]}$ 。 现有研究表明普遍认为 ${ }^{[4-9]} \mathrm{Fe}-\mathrm{Al}$ 脆性金属间化合物厚 度控制在 $10 \mu \mathrm{m}$ 以内可保证其力学性能。近年来提出 的熔钎焊方法能够将金属间化合物厚度控制在极低 的水平, 是一种极具潜力的钢/铝焊接方法 ${ }^{[5,7,10]}$ 。大量 
试验结果也证明, 熔钎焊可以获得较好的接头力学性 能, 特别是高能量密度的激光熔钎焊技术。然而, 激 光熔钎焊技术一直存在液态金属铝在钢表面润湿铺展 性差的问题, 导致激光熔钎焊一直未能实际应用。

液态铝在通常情况下几乎无法在钢表面直接实 现润湿铺展, 无辅助措施下难以焊接, 因此润湿铺 展也是钢/铝异种金属激光熔钎焊面临的主要问题 之一。当前主要采用三种方法解决该问题, 即采用 药芯焊丝 ${ }^{[11]}$ 、预涂钎剂 ${ }^{[12]}$ 和钢表面镀锌 ${ }^{[13-14]}$ 。然而 这些方法在应用的过程中受到了极大的限制。首先, 当前并不是所有的焊丝都是药芯焊丝且难以应用无 丝焊接的情况。其次, 虽然预涂钎剂方法能够实现 非药芯焊丝熔钎焊, 但是其生产效率和过程的稳定 性受到了极大的限制。再者, 所有接头均采用镀锌 层的方式辅助润湿并不具备现实意义。因此, 开发 出一种适应性强与过程稳定的解决润湿铺展方法是 钢/铝异种金属激光熔钎焊方法面临的主要问题。

基于上述的问题, 文中提出采用气载钎剂方式 辅助激光熔钎焊的润湿铺展, 通过专用的送粉器把 钎剂与保护气体一起均匀的送入到焊接熔池中, 使 液态钎剂覆盖在液态金属表面。研究了线能量对于 润湿角、接头力学性能的影响, 同时分析了接头界 面的连接特性。

\section{1 试验}

\section{1 试验材料}

试验母材是 316 不锈钢和 5052 铝合金, 其主要 成分见表 1 , 母材规格均为 $2 \mathrm{~mm} \times 50 \mathrm{~mm} \times 100 \mathrm{~mm}$ 。 焊丝为 $\phi 1.2 \mathrm{~mm}$ 的 $5356(\mathrm{Al}-5 \mathrm{Mg})$, 成分含量如表 2 所示。不锈钢在焊前采用丙酮清洗表面油污及杂质。 5052 铝合金板先浸在 $10 \%$ 的 $\mathrm{NaOH}$ 溶液中(温度为 $30 \sim 50{ }^{\circ} \mathrm{C}$ ), 浸洗 2 3 min, 浸后产生大量气泡, 并形成一层黑色薄膜, 然后用水将铝板表面的碱液 冲洗干净。再将铝板浸入 $30 \%$ 的硝酸溶液中, 浸洗 $1 \mathrm{~min}$ 后取出, 待表面呈现为白色且光亮, 用水将表 面残留的硝酸溶液冲洗干净并用吹风机吹干。

表 1 试验材料的化学成分(质量分数) $\%$

\begin{tabular}{|c|c|c|c|c|c|c|c|}
\hline 材料 & $\mathrm{C}$ & $\mathrm{Si}$ & $\mathrm{Fe}$ & Mo & $\mathrm{Ni}$ & $\mathrm{Mg}$ & $\mathrm{Cr}$ \\
\hline 316 & $\leqslant 0.08$ & $\leqslant 1.0$ & 余量 & $\leqslant 2.0$ & $\begin{array}{c}10.0 \sim \\
14.0\end{array}$ & - & $\begin{array}{c}16.0 \sim \\
18.0\end{array}$ \\
\hline 5052 & - & $\leqslant 0.25$ & $\leqslant 0.4$ & - & - & $\begin{array}{c}2.2 \sim \\
2.8\end{array}$ & $\begin{array}{c}0.15 \sim ~ \\
0.35\end{array}$ \\
\hline
\end{tabular}

表 25456 药芯焊丝成分(质量分数) $\%$

\begin{tabular}{ccccccccc}
\hline 材料 & $\mathrm{Si}$ & $\mathrm{Fe}$ & $\mathrm{Mn}$ & $\mathrm{Mg}$ & $\mathrm{Zn}$ & $\mathrm{Cr}$ & $\mathrm{Al}$ \\
\hline \multirow{2}{*}{5356} & \multirow{2}{*}{$\leqslant 0.25$} & $\leqslant 0.40$ & $0.05 \sim$ & $4.50 \sim$ & & $0.05 \sim$ & \multirow{2}{*}{ 余量 } \\
& & 0.20 & 5.50 & & 0.20 & \\
\hline
\end{tabular}

\section{2 试验方法}

试验采用的设备是 IPG LYS-400 光纤激光器和 KUKA KR-60 焊接机器人。焊接过程如图 1 所示, 采取铝上钢下的搭接形式, 送粉嘴和送丝嘴分列激 光的两侧, 它们与工件的角度大约为 $30^{\circ}$, 激光位 于中心。Noclock 钎剂用喷雾造粒的方法制成易于 流动的颗粒状, 利用专用的送粉器把钎剂与保护气 体一起均匀的送入焊接熔池中。先期探索得到激光 熔钎焊工艺参数范围为激光功率 $1800 \sim 3000 \mathrm{~W}$, 焊接速度 $0.5 \sim 1.0 \mathrm{~m} / \mathrm{min}$, 送丝速度维持在 3.5 $\mathrm{m} / \mathrm{min}$ 。光斑直径为 $2 \sim 3 \mathrm{~mm}$, 离焦量为 $40 \mathrm{~mm}$ 。 采用上述区间内不同工艺参数焊接 316 不锈钢/5052 铝合金。

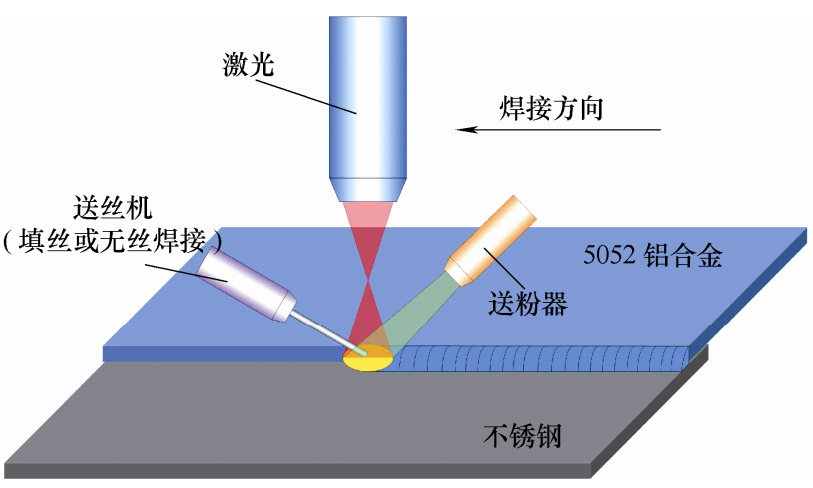

图 1 工艺焊接示意图

将激光焊接的试样沿垂直焊缝的方向线切割, 制成 $10 \mathrm{~mm} \times 5 \mathrm{~mm}$ 的小试样。采用标准的金相试样 制备方法进行打磨抛光，满足要求后对金相试样用 Keller 试剂进行腐蚀, 金相试样腐蚀完成后用水冲 洗以去除表面残留的腐蚀液, 然后用吹风机将其吹 干，吹干后的金相试样即可在光学显微镜下观察金 相并拍照。

采用 JSM-6510A 扫描电子显微镜 (Scanning electron microscope, SEM)对焊接接头的微观形貌及 拉伸断口形貌进行形貌分析, 对焊缝的局部区域进 行能谱分析(Energy dispersive spectrometer, EDS), 以确定接头断面处金属间化合物的成分组成。

焊接得到的试样进行切割, 加工成 $100 \mathrm{~mm} \times 25$ $\mathrm{mm}$ 的拉伸试样, 焊缝位于拉伸试样中部, 对接头 试样进行拉伸, 速率选用 $1.5 \mathrm{~mm} / \mathrm{min}$, 试样被拉断 时记录其峰值, 再根据断裂处的承载截面积计算出 抗拉强度。

\section{2 焊接线能量对接头润湿角的影响}

激光功率、焊接速度主要影响焊接线能量的变 化, 线能量的变化直接影响熔化钎料和固态钢表面 
的温度, 进而影响熔化金属表面张力和黏度, 这是 影响液态金属在钢表面润湿铺展的主要因素。润湿 角是评定润湿性的一个直观因素, 润湿角的测量标 准如图 2 所示, 其具体数值通过所制备的金相试样 在扫描电镜下测得, 润湿角越小, 液态金属在钢表 面的润湿铺展性越好。

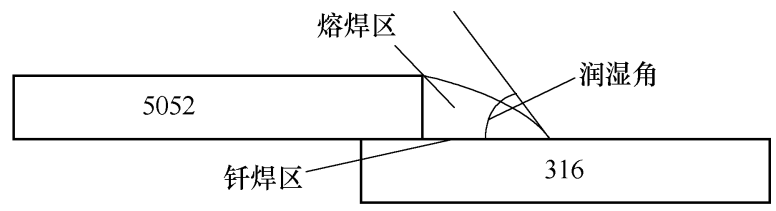

图 2 搭接润湿角的测量

激光功率和焊接速度对于接头润湿角都有显着 影响。改变任何一个工艺参数都是改变焊接线能量, 从而影响接头的润湿铺展。因此, 研究焊接线能量 对润湿角的影响更加合适。焊接线能量的计算方式 如下

$$
Q=\frac{3 P}{50 v}
$$

式中, $Q$ 为线能量, $\mathrm{J} / \mathrm{mm} ; P$ 为激光功率, $\mathrm{W} ; v$ 为焊接速度, $\mathrm{m} / \mathrm{min}$ 。

图 3 所示为填丝搭接激光熔钎焊通过上式计算 得到的不同焊接线能量下润湿角的变化。从图中可 以看出随着焊接线能量的变化, 液态金属在钢表 面的润湿角呈先降低后增加的趋势。当焊接线能 量较低时, 搭接接头的单位热输入量偏低, 熔化 金属表面张力和黏性较大，其在钢表面的润湿铺 展性较差, 润湿角偏大; 随着焊接线能量的增加, 熔化金属表面张力和粘性较低, 其在钢表面润湿 铺展性更好, 润湿角变小; 但是当焊接线能量过 高时, 接头单位热输入量偏高, 导致钎剂部分烧 损而不能有效去除液态金属表面的氧化膜，润湿 角较大, 即液态金属在钢表面的润湿铺展性差。 当焊接线能量为 $360 \mathrm{~J} / \mathrm{mm}$ 时, 接头润湿角达到最 小值 $37^{\circ}$ 。

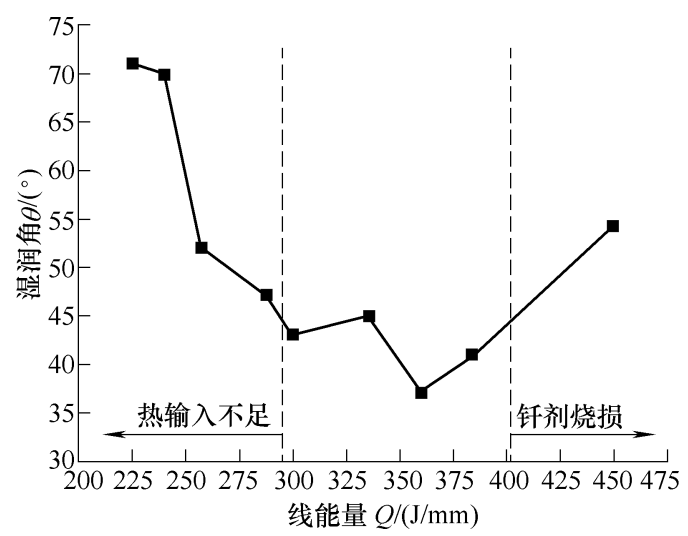

图 3 填丝激光熔钎焊中润湿角随线能量变化
图 4 为无丝搭接激光熔钎焊中润湿角随焊接线 能量的变化, 从图中可以看出, 随着焊接线能量的 升高, 液态铝在钢表面的润湿角先降低后升高, 这 和填丝搭接激光熔钎焊润湿角变化规律一致。当焊 接线能量为 $264 \mathrm{~J} / \mathrm{mm}$, 润湿角达到最小值 $18.8^{\circ}$, 液态铝在钢表面的润湿铺展性达到最佳。

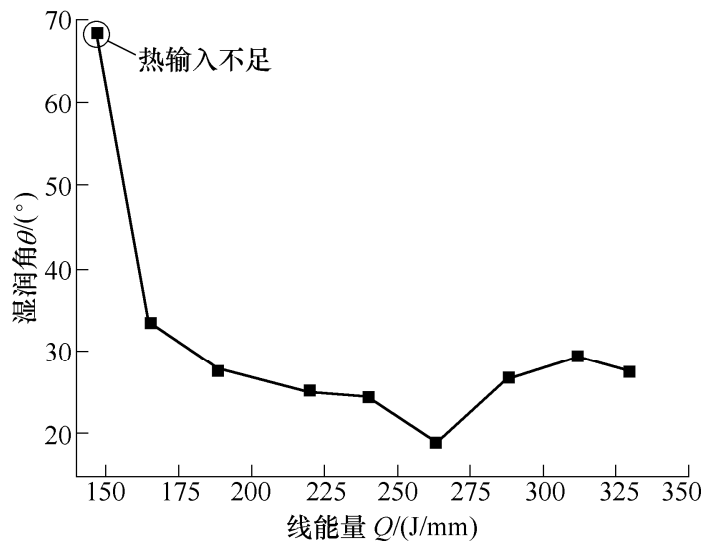

图 4 无丝激光熔钎焊中润湿角随线能量变化

通过对比分析不同线能量对填丝和无丝激光 熔钎焊搭接润湿角变化可以发现：填丝搭接激光 熔钎焊中当焊接线能量为 $360 \mathrm{~J} / \mathrm{mm}$ 时, 即工艺参 数为送丝速度 $3.5 \mathrm{~m} / \mathrm{min}$ 、焊接速度 $0.5 \mathrm{~m} / \mathrm{min}$ 、激 光功率 $3000 \mathrm{~W}$ 、离焦量 $40 \mathrm{~mm}$ 、保护气体流量为 $3 \mathrm{~L} / \mathrm{min}$, 润湿角达到最小值 $37^{\circ}$; 无丝搭接激光熔 钎焊中当焊接线能量为 $264 \mathrm{~J} / \mathrm{mm}$, 即工艺参数为焊接 速度 $0.5 \mathrm{~m} / \mathrm{min}$ 、激光功率 $2200 \mathrm{~W}$ 、离焦量 $+40 \mathrm{~mm}$ 、 保护气体流量为 $3 \mathrm{~L} / \mathrm{min}$ 时, 润湿角达到最小值 $18.8^{\circ}$ 。 填丝搭接熔钎焊中液态金属润湿铺展性达到最佳的 线能量比无丝搭接的高, 这主要是因为填丝焊接中 激光能量一部分用来熔化铝母材和加热不锈钢, 还 需要另一部分用来熔化焊丝, 而无丝焊接中能量只 用来熔化母材和加热不锈钢, 所以填丝焊消耗能量 比无丝焊高, 焊接线能量相对较高。无丝焊最小润 湿角比填丝焊的小，这主要是因为填丝焊液态金属 中有一部分为熔化的焊丝, 液相量相对较大, 所以 其润湿角相对较大。

\section{3 焊接线能量对接头力学性能的影响}

填丝搭接激光熔钎焊不同焊接线能量下接头力 学性能的变化如图 5 所示。从图 5 可知, 随着焊接 线能量的增加, 接头的拉伸强度呈先增加后降低的 趋势。随着线能量的增加，液态金属在钢表面的润 湿铺展性变好, 接头强度随之增加; 焊接线能量过 大时, 单位热输入量过高, 钎剂大量挥发, 液态金 属润湿铺展性变差, 不锈钢和铝合金界面生成大量 
金属间化合物, 从而导致接头拉伸强度的降低。当 线能量为 $360 \mathrm{~J} / \mathrm{mm}$ 时, 接头拉伸强度达到最大值 $132.8 \mathrm{MPa}$ 。

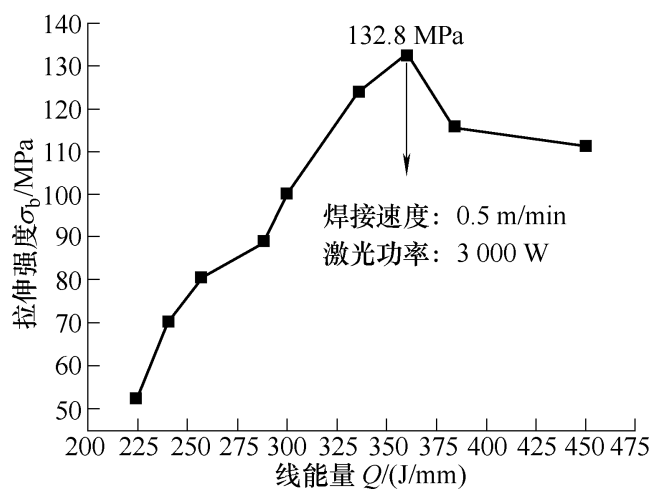

图 5 填丝搭接激光熔钎焊接头拉伸强度随线能量的变化

无丝搭接激光熔钎焊不同焊接线能量下拉伸强 度的变化如图 6 所示。从图 6 可以看出, 随焊接线 能量的增加, 接头拉伸强度呈先增加后减少的趋势。 当焊接线能量低于 $264 \mathrm{~J} / \mathrm{mm}$ 时, 拉伸强度随线能量 的增加近乎呈直线上升的趋势; 当焊接线能量高于 $264 \mathrm{~J} / \mathrm{mm}$ 时, 随线能量的增加接头强度变化不大; 焊接线能量为 $264 \mathrm{~J} / \mathrm{mm}$, 接头强度达到最大值 109.2 $\mathrm{MPa}$ 。

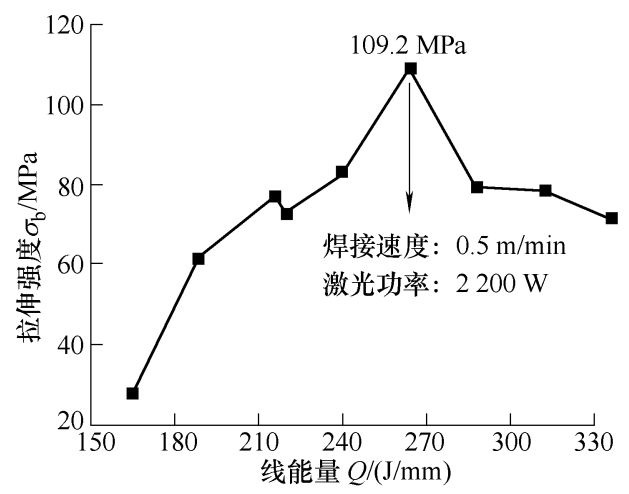

图 6 无丝搭接激光熔钎焊接头拉伸强度随线能量变化

以上试验结果的分析可以看出, 接头拉伸强 度受焊接工艺参数变化影响较大, 焊接工艺参数 的改变主要影响焊接线能量的变化, 线能量的变 化直接影响液态金属在钢表面的润湿铺展性, 进 而影响焊接接头的力学性能。填丝搭接激光熔钎 焊中, 当激光功率为 $3000 \mathrm{~W}$, 焊接速度为 0.5 $\mathrm{m} / \mathrm{min}$, 即焊接线能量为 $360 \mathrm{~J} / \mathrm{mm}$ 时, 搭接接头 的润湿角最小, 接头拉伸强度达到 $132.8 \mathrm{MPa}$; 无 丝搭接激光熔钎焊中, 当激光功率为 $2200 \mathrm{~W}$, 焊 接速度为 $0.5 \mathrm{~m} / \mathrm{min}$, 即焊接线能量为 $264 \mathrm{~J} / \mathrm{mm}$, 搭接接头润湿角达到最小, 接头拉伸强度为 109.2 $\mathrm{MPa}$ 。从最佳工艺参数下力学性能来看, 填丝搭 接接头强度明显高于无丝搭接接头。在焊接过程
中, 填充焊丝有利于提高接头的力学性能, 这主 要是因采用填充焊丝可以有效增加液相量, 扩大 钢/铝界面有效连接宽度。此外, 在焊丝中的合金 元素与铝合金相比具有一定的差异, 这种差异会 对钢/铝界面产生一定的影响, 进一步会影响接头 力学性能。

\section{4 激光熔钎焊接头组织分析}

从对不锈钢/铝合金填丝激光熔钎焊和无丝激 光熔钎焊润湿角和接头力学性能来看: 填丝激光熔 钎焊中当激光功率 $3000 \mathrm{~W}$ 、送丝速度 $3.5 \mathrm{~m} / \mathrm{min}$ 、 焊接速度 $0.5 \mathrm{~m} / \mathrm{min}$ 、离焦量 $+40 \mathrm{~mm}$ 、保护气体流 量为 $3 \mathrm{~L} / \mathrm{min}$ 时得到的搭接接头无论接头形貌、润 湿铺展性和接头力学性能均达到相对较佳值; 无丝 激光熔钎焊中最佳工艺参数为激光功率 $2200 \mathrm{~W}$ 、 焊接速度 $0.5 \mathrm{~m} / \mathrm{min}$, 离焦量 $40 \mathrm{~mm}$ 、保护气体流量 为 $3 \mathrm{~L} / \mathrm{min}$ 。因此, 接下来分别对填丝和无丝最佳工 艺参数下得到的接头显微组织进行分析, 对比填丝 与无丝接头组织的差异性。

图 7a 所示钢/铝焊缝成形较好。图 7b 为填丝激 光熔钎焊典型接头宏观截面形貌, 从图中可知焊缝 界面钢侧几乎未熔化, 铝合金侧靠近热源熔化, 液态金属在气载式钎剂辅助作用下能够在不锈钢 表面有效润湿铺展, 焊缝根部靠近铝母材侧有明 显的孔洞, 主要是因为该处远离焊接热源, 温度 太低导致熔化金属在不锈钢表面的润湿铺展性较 差, 因此有孔洞的产生。接头主要由钎焊连接区 和熔焊连接区两部分组成, 钎焊连接区是激光熔 钎焊的薄弱环节, 显微组织的分析主要是针对钎 焊区域。按照激光熔钎焊热源分布, 钎焊界面分 为 $\mathrm{A} 、 \mathrm{~B} 、 \mathrm{C}$ 三个区域。

图 7b 中钢和焊缝界面 A、B、C 区域的微观组 织如图 7c、7d、7e 所示, 区域 $\mathrm{A}$ 的界面金属间化 合物(Intermetallic compounds, IMCs)厚度较薄, 大 约为 $4 \mu \mathrm{m}$, IMCs 与焊缝和钢均呈锯齿状结合; 区域 B 的 IMCs 最厚, 达到 $7 \mu \mathrm{m}$ 以上, IMCs 靠 近焊缝侧呈长条状向焊缝中生长, 靠近钢侧呈平 面状; 区域 $\mathrm{C}$ 的 IMCs 厚度为 $3 \sim 4 \mu \mathrm{m}$, 靠近焊缝 呈条块状, 而靠近钢侧呈平面状, 并有裂纹产生。 分析原因: B 区域位于激光焊接过程中的能量中 心, 峰值温度较高, 界面反应时间均较长, IMCs 厚度最高, 呈长条状向焊缝中生长; 区域 $\mathrm{A}$ 与 $\mathrm{C}$ 距离激光能量中心有一定距离, 峰值温度较低, 界面反应时间较短, IMCs 的厚度均较薄, 此区域 热输入低, 润湿铺展最差, 也是裂纹产生的原因。 


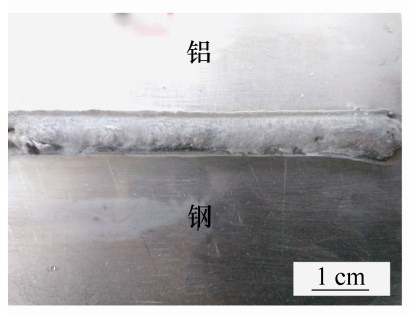

(a) 填丝接头焊缝成形

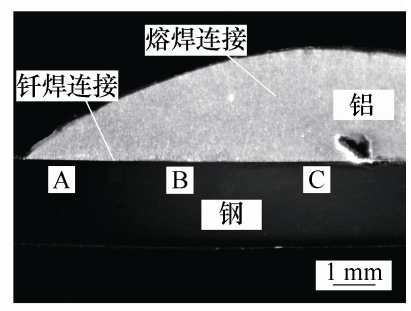

(b) 接头横截面形貌

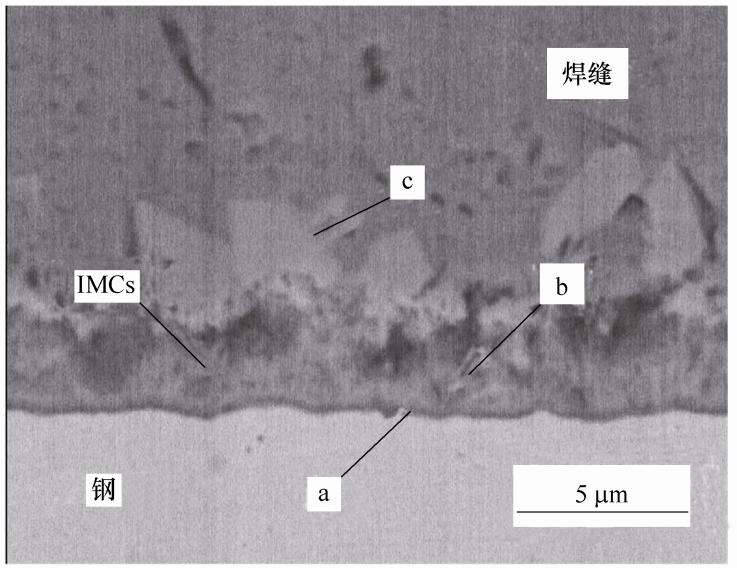

(c) A 区放大图

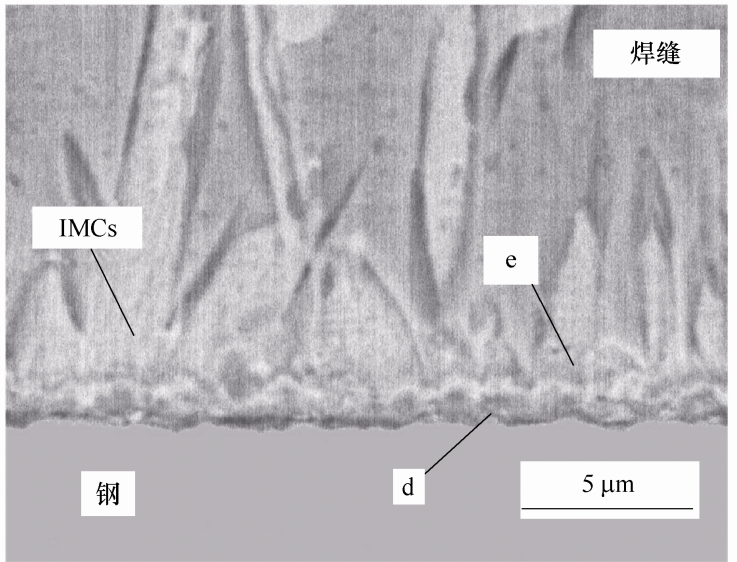

(d) B 区放大图

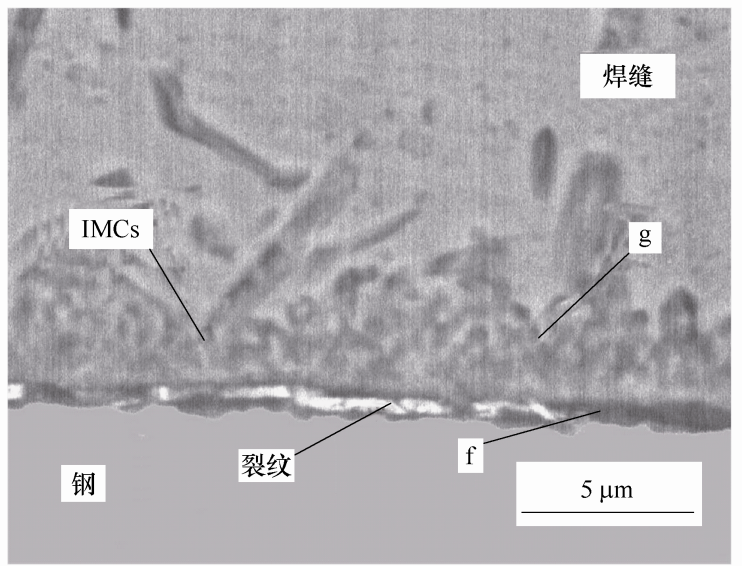

(e) C 区放大图

图 7 填丝激光熔钎焊典型接头组织

对图 7 中的 IMCs 进行 EDS 分析, 结果列于表 3 。从表中可以看到从钢到焊缝, $\mathrm{Al}$ 含量升高, $\mathrm{Fe}$ 含量降低, 靠近钢侧的( $a 、 d 、 f 区$ 区域)元素原子含量: Fe: $69.69 \% \sim 72.14 \%, \mathrm{Al}+\mathrm{Ni}: \quad 27.86 \% \sim 30.31 \%$,
分析该层 $\mathrm{IMCs}$ 为 $\mathrm{Fe}_{2} \mathrm{Al}_{5}$ 相; 靠近焊缝侧(b、e、g 区 域)的元素原子含量: $\mathrm{Al}: 76.04 \% \sim 77.44 \%, \mathrm{Fe}+\mathrm{Ni}$ : $22.56 \% \sim 23.97 \%$, 分析其 $\mathrm{IMCs}$ 为 $\mathrm{FeAl}_{3}$ 相, $\mathrm{c}$ 区域 元素含量主要为 $\mathrm{Al}$, 分析其应是 $\mathrm{Fe}$ 在 $\mathrm{Al}$ 中形成的 固溶体: $\alpha-\mathrm{Al}$ 。

表 3 钢侧界面各相元素组成

$\%$

\begin{tabular}{cccc}
\hline \multirow{2}{*}{ 位置 } & \multicolumn{3}{c}{ 元素 } \\
\cline { 2 - 4 } & $\mathrm{Al}$ & $\mathrm{Fe}$ & $\mathrm{Ni}$ \\
\hline $\mathrm{a}$ & 73.16 & 17.04 & 9.80 \\
$\mathrm{~b}$ & 77.00 & 15.00 & 8.00 \\
$\mathrm{c}$ & 95.33 & 4.67 & - \\
$\mathrm{d}$ & 72.14 & 27.86 & - \\
$\mathrm{e}$ & 77.44 & 22.56 & - \\
$\mathrm{f}$ & 69.69 & 16.43 & 13.88 \\
$\mathrm{~g}$ & 76.04 & 17.97 & 6.00 \\
\hline
\end{tabular}

分析 IMCs 不同相的形成原因: 主要是焊接过 程中, 固态钢和液态钎料相互作用, 固态钢部分溶 解, 钢中的 $\mathrm{Fe}$ 原子进入到焊接熔池中，同时 $\mathrm{Al}$ 原 子也向钢中扩散, 靠近钢侧的 $\mathrm{Fe}$ 原子含量较高, 最 终通过反应扩散形成 $\mathrm{Fe}_{2} \mathrm{Al}_{5}$ 相, 靠近焊缝侧的 $\mathrm{Al}$ 原子含量较高, 最终形成 $\mathrm{FeAl}_{3}$ 相。CHEN 等 ${ }^{[2]}$ 通 过研究 $\mathrm{Fe} / \mathrm{Al}$ 固液界面反应得出结论为: 在温度较 低时, 界面 $\mathrm{FeAl}_{3}$ 的形成受到反应扩散与冷却析出 机制的联合控制, 在温度较高时, 界面 $\mathrm{FeAl}_{3}$ 的形 成机理仅为冷却析出。

图 8a 为无丝焊接接头宏观形貌, 焊缝成形良 好。图 $8 \mathrm{~b}$ 所示为无丝搭接激光熔钎焊典型接头宏观 截面形貌, 从图中可知焊缝界面钢侧几乎未熔化, 铝合金侧靠近热源熔化, 液态金属在气载式钎剂辅 助作用下能够在钢表面有效润湿铺展, 焊缝根部靠 近铝母材侧有明显的孔洞, 接头主要由钎焊连接区 和熔焊连接区两部分组成, 钎焊连接区是激光熔钎 焊的薄弱环节，显微组织的分析主要是针对钎焊区 域。按照激光熔钎焊热源分布, 钎焊界面分为 $\mathrm{A}$ 、 B、C 三个区域。

图 $8 b$ 中钢和焊缝界面 A、B、C 区域的微观组 织如图 8c、8d、8e 中所示，从图中可以看到 $\mathrm{A}$ 区、 $\mathrm{C}$ 区中 IMCs 呈片状生长, 其厚度约为 $4 \mu \mathrm{m}$, IMCs 和钢界面呈锯齿状结合; B 区中 IMCs 的形貌和厚 度和 A、C 区有很大差别, 部分 IMCs 呈针块状散 布于焊缝中, IMCs 与钢的界面由裂纹存在。这主要 是因为激光热源熔化铝母材过程中热源分布不均, 中心区域温度相对较高, IMCs 的生长较快, 部分呈 现长条状, 并且因温度过高, IMCs 与钢的界面发生 开裂; 焊缝两侧因远离热源, 温度相对较低, IMCs 的生长减慢, 与钢的界面结合也比较牢固。 


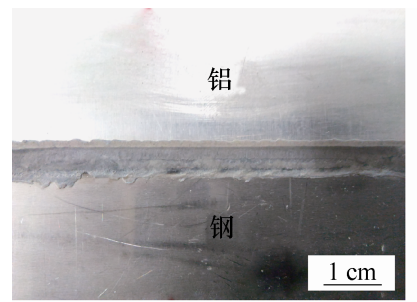

(a) 无丝接头焊缝成形

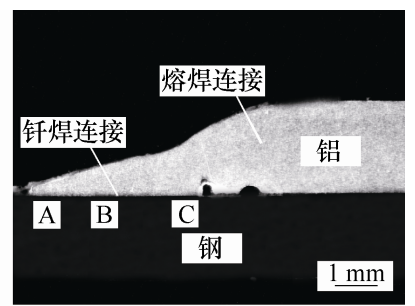

(b) 接头横截面形貌

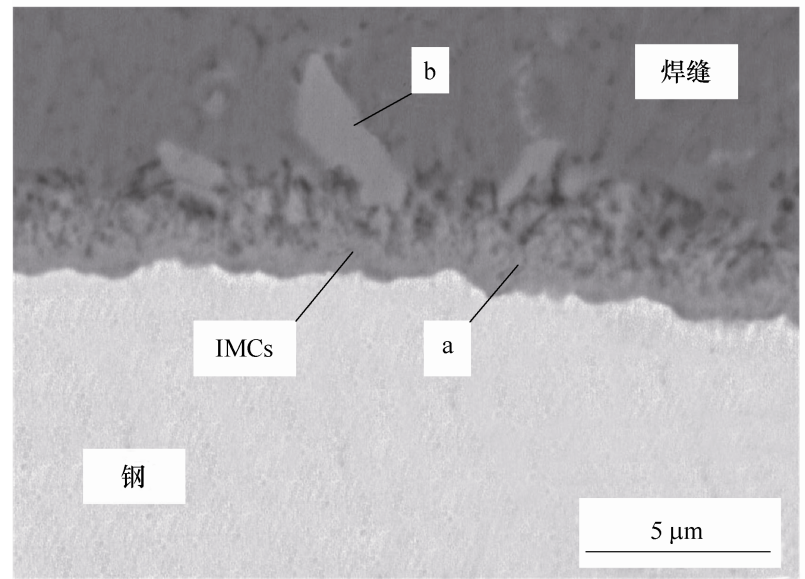

(c) A 区放大图

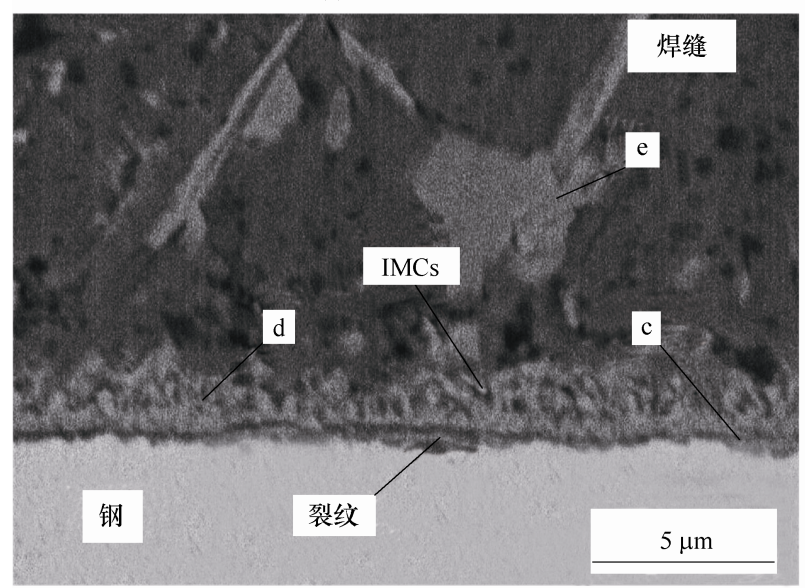

(d) B 区放大图

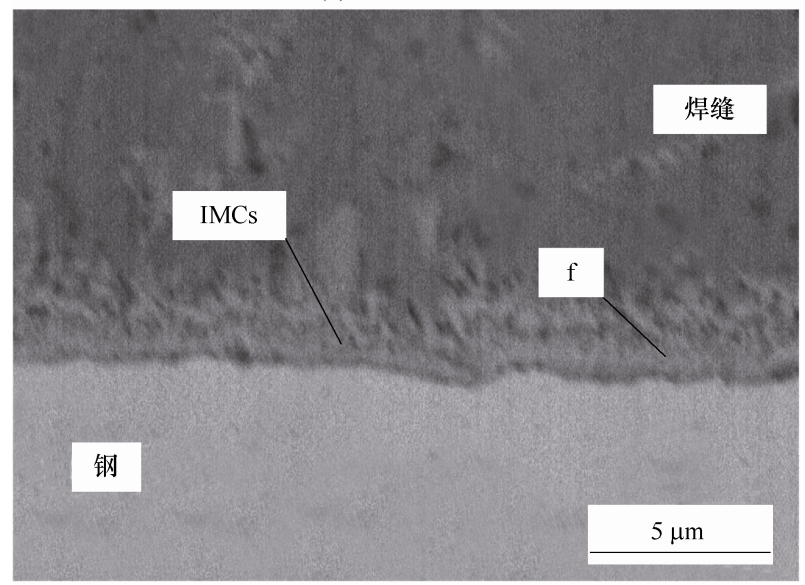

(e) C 区放大图

图 8 无丝激光熔钎焊典型接头组织

对图 8 中 IMCs 的成分进行 EDS 分析, 结果如 表 4 所示。从表中可以看到, 从钢到焊缝, $\mathrm{Al}$ 含量 升高, 钢含量降低。钢侧的 $\mathrm{c}$ 点 $\mathrm{Al}$ 含量相对较低,
$\mathrm{Fe}$ 含量相对较高, 分析该 IMCs 为 $(\mathrm{Fe}, \mathrm{Cr})_{2} \mathrm{Al}_{5}$, 即 固溶一定 $\mathrm{Cr}$ 元素的 $\mathrm{Fe}_{2} \mathrm{Al}_{5}$ 相; 靠近焊缝侧( $\mathrm{a} 、 \mathrm{~d}$ 、 f)元素含量： $\mathrm{Al}: \quad 76.54 \% \sim 78.37 \%, \mathrm{Fe}: 17.04 \%$ $20.84 \%, \mathrm{Cr}: 2.38 \% \sim 4.73 \%$, 分析 $\mathrm{IMCs}$ 为 $\mathrm{FeAl}_{3}$; 散布于焊缝中呈针块状 (b、e)的元素含量： $\mathrm{Al}$ : $85.21 \% \sim 85.77 \%$, Fe: $4.22 \% \sim 4.34 \%$, Cr: $10.01 \% \sim$ $10.44 \%$, 分析该化合物为 $\mathrm{Fe}$ 在铝中形成的 $\alpha-\mathrm{Al}$ 固 溶体。表明焊接过程中, 固态钢和液态铝相互作用, 钢侧的 $\mathrm{Fe} 、 \mathrm{Cr}$ 向焊缝中扩散, 同时焊缝中的 $\mathrm{Al}$ 向 钢侧扩散, 靠近钢侧因 $\mathrm{Fe} 、 \mathrm{Cr}$ 含量相对较高, 生成 的 IMCs 为 $\mathrm{Fe}_{2} \mathrm{Al}_{5}$ 相, 靠近焊缝处 $\mathrm{Al}$ 含量相对较高, 生成 $\mathrm{FeAl}_{3}$ 相。焊缝中游离分布针块状的金属间化 合物主要高温时扩散进入到铝液中的 $\mathrm{Fe}$ 原子, 焊接 过程中熔池流动也加剧了 $\mathrm{Fe}$ 原子的迁移。降温过程 中原子在铝中极限溶解度降低, 在冷却时以 $\mathrm{FeAl}_{3}$ 金属间化合物的形式析出。

表 4 IMCs 的元素组成

$\%$

\begin{tabular}{cccc}
\hline \multirow{2}{*}{ 位置 } & \multicolumn{3}{c}{ 元素 } \\
\cline { 2 - 4 } & $\mathrm{Al}$ & $\mathrm{Fe}$ & $\mathrm{Cr}$ \\
\hline $\mathrm{a}$ & 78.37 & 17.04 & 4.59 \\
$\mathrm{~b}$ & 85.77 & 4.22 & 10.01 \\
$\mathrm{c}$ & 70.39 & 23.70 & 5.91 \\
$\mathrm{~d}$ & 76.78 & 20.84 & 2.38 \\
$\mathrm{e}$ & 85.21 & 4.34 & 10.44 \\
$\mathrm{f}$ & 76.54 & 18.73 & 4.73 \\
\hline
\end{tabular}

\section{5 结论}

（1）对于不锈钢/铝合金异种金属填丝激光熔钎 焊, 随焊接线能量的增加, 即激光功率增加或焊接 速度降低, 液态金属在不锈钢表面的润湿铺展先变 好后变差。焊接线能量为 $360 \mathrm{~J} / \mathrm{mm}$ 时, 即工艺参数 为送丝速度 $3.5 \mathrm{~m} / \mathrm{min}$ 、焊接速度 $0.5 \mathrm{~m} / \mathrm{min}$ 、激光 功率 $3000 \mathrm{~W}$ 时润湿角达到最小值 $37^{\circ}$; 对于无丝 搭接激光熔钎焊, 随焊接线能量的增加, 即激光功 率增加或焊接速度降低, 液态金属在不锈钢表面的 润湿铺展也是先变好后变差, 焊接线能量为 264 $\mathrm{J} / \mathrm{mm}$ 时, 即工艺参数为焊接速度 $0.5 \mathrm{~m} / \mathrm{min}$ 、激光 功率 $2200 \mathrm{~W}$ 时润湿角达到最小值 $18.8^{\circ}$ 。

(2) 对于不锈钢/铝合金异种金属填丝激光熔钎 焊, 随焊接线能量的增加, 接头拉伸强度呈先增加 后降低的趋势, 当激光功率为 $3000 \mathrm{~W}$, 送丝速度 $3.5 \mathrm{~m} / \mathrm{min}$, 焊接速度为 $0.5 \mathrm{~m} / \mathrm{min}$, 即焊接线能量为 $360 \mathrm{~J} / \mathrm{mm}$ 时, 搭接接头的润湿角最小, 接头拉伸强 度达到 $132.8 \mathrm{MPa}$; 对于无丝搭接激光熔钎焊, 接 头拉伸强度随焊接线能量的增加也是先增加后降 
低, 当激光功率为 $2200 \mathrm{~W}$, 焊接速度为 $0.5 \mathrm{~m} / \mathrm{min}$, 即焊接线能量为 $264 \mathrm{~J} / \mathrm{mm}$, 搭接接头润湿角达到最 小，接头拉伸强度为 $109.2 \mathrm{MPa}$ 。

(3) 不锈钢/铝合金异种金属填丝激光熔钎煌, 钢/焊缝界面不同位置的 IMCs 厚度和形貌不同, 中 心位置因峰值温度高, IMCs 呈长条状, 厚度达到 7 $\mu \mathrm{m}$ 以上; 顶部位置峰值温度相对较低, IMCs 呈锯 齿状, 厚度为 $4 \mu \mathrm{m}$; 根部位置因远离焊接熔池, 钎 剂不能有效送达, 再加上峰值温度较低, 液态钎料 不能有效润湿铺展, 因此存在未焊合的孔洞。填丝 和无丝熔钎焊中界面 IMCs 靠近焊㖓侧为 $\mathrm{FeAl}_{3}$, 靠 近钢侧为 $\mathrm{Fe}_{2} \mathrm{Al}_{5}$, 焊缝中游离分布呈块状的化合物 为 $\mathrm{Fe}$ 在铝中形成的 $\mathrm{FeAl}_{3}$ 。

\section{参 考 文 献}

[1] CHEN Shuhai, YANG Dawei, YANG Jian, et al. Nanoscale structures of the interfacial reaction layers between molten aluminium and solid steel based on thermophysical simulations[J]. Journal of Alloy and Compounds, 2018, 739: 184-189.

[2] CHEN Shuhai, YANG Dongdong, ZHANG Minxin, et al. Interaction between the growth and dissolution of intermetallic compounds in the interfacial reaction between solid iron and liquid aluminum[J]. Metallurgical and Materials Transactions A, 2016, 47A: 5088-5100.

[3] 龙江启, 兰凤崇, 陈吉清. 车身轻量化与钢铝一体化结 构新技术的研究进展 [J]. 机械工程学报, 2008, 44(6): 27-35.

LONG Jiangqi, LAN Fengchong, CHEN Jiqing. New technology of lightweight and steel-aluminum hybrid structure car body[J]. Chinese Journal of Mechanical Engineering, 2008, 44(6): 27-35.

[4] SPRINGER H, KOSTKA A, PAYTON E J , et al. On the formation and growth of intermetallic phases during interdiffusion between low-carbon steel and aluminum alloys[J]. Acta Materialia, 2011, 59(4): 1586-1600.

[5] 石玗, 温俊霞, 黄健康, 等. 基于旁路耦合电弧的铝钢 MIG 熔钎焊研究 $[\mathrm{J}]$. 机械工程学报, 2011，47(16): 25-29.

SHI Yu, WEN Junxia, HUANG Jiankang, et al. Study on DE-GMAW MIG-brazing method for bonding steel with aluminum $[\mathrm{J}]$. Chinese Journal of Mechanical Engineering, 2011, 47(16): 25-29.

[6] JAIVINDRA S, KANWER S A, DIESH K S. Dissimilar MIG-CMT weld-brazing of aluminum to steel: A review[J]. Journal of Alloy and Compounds, 2019, 783: 753-764.

[7] 陈树海, 黄继华, 陈彦宾, 等. 异种合金激光熔钎焊技 术的现状与展望[J]. 焊接, 2011, 4：27-31.

CHEN Shuhai, HUANG Jihua, CHEN Yanbin. The present situation and prospect of laser weld-brazing technology of dissimilar alloys[J]. Welding and Joining, 2011, 4: 27-31.

[8] MASOUD M, NIMA Y, YANG Guang, et al. Effect of dual laser beam on dissimilar welding-brazing of aluminum to galvanized steel[J]. Optics and Laser Technology, 2018, 98(1): 214-228.

[9] WAGNER F, ZERNER I, KREIMEYER M, SEEFELD T, et al. Characterization and properties of dissimilar metal combinations of $\mathrm{Fe} / \mathrm{Al}$ and $\mathrm{Ti} / \mathrm{Al}$-sheet materials[C/CD]//Proc. ICALEO'01 (CD-ROM), 2001.

[10] 林三宝, 宋建岭, 杨春利. 铝合金/不锈钢铇极氩弧熔钎焊接头界面层的微观结构分析 [J]. 金属学报, 2009, 45(10): 1211-1216.

LIN Sanbao, SONG Jianling, YANG Chunli, et al. Microstructure analysis of interfacial layer with tungsten inert gas welding-brazing joint of aluminum/stainless steel[J]. Acta Metallurgica Sinica, 2009, 45(10): 1211-1216.

[11] DONG Honggang, HU Wenjin, ZHANG Xuchao. Detachment of interfacial layers during arc-brazing of aluminum alloy to carbon steel with filler wire[J]. Transactions of Nonferrous Metals Society of China, 2013, 23(6): 1583-1588.

[12] 宋建岭, 林三宝, 杨春利, 等. 特种钎剂辅助铝/钢异种 合金 TIG 熔-钎焊 [J]. 焊接学报, 2010, 31(2)：45-48.

SONG Jianling, LIN Sanbao, YANG Chunli, et al. TIG welding-brazing of special flux assisted aluminum-steel dissimilar alloys[J]. Transactions of the China Welding Institution, 2010, 31(2): 45-48.

[13] 檀财旺, 梅长兴, 李俐群, 等. 镁/镀锌钢异种合金单、 双光束激光熔钎焊特性 [J]. 中国有色金属学报, 2012(6): 1577-1585.

TAN Caiwang, MEI Changxin, LI Liqun, et al. Single and dual beam laser welding-brazing characteristics of magnesium/zinc-coated steel dissimilar alloys. The Chinese Journal of Nonferrous Metals, 2012(6): 1577-1585.

[14] 雷振, 秦国梁, 林尚扬, 等. 基于激光-MIG 复合热源 的 $5 \mathrm{~A} 02$ 铝合金/镀锌钢熔一钎焊 [J]. 机械工程学报, 2009, 45(3): 94-98.

LEI Zhen, QIN Guoliang, LIN Shangyang, et al. Fusion-brazing joining for dissimilar metals between 5A02 aluminium alloy and Zinc-coated steel based on laser-MIG hybrid welding[J]. Journal of Mechanical Engineering, 2009, 45(3): 94-98.

作者简介: 喻高扬, 男, 1994 年出生, 博士研究生。主要研究方向钢/ 铝异种金属连接。

E-mail: yugaoyang66@163.com

陈树海(通信作者), 男, 1979 年出生, 博士, 教授。主要研究方向为异 种材料焊接新技术、现代激光焊接技术、材料连接过程界面行为。

E-mail: shchen@mater.ustb.edu.cn 